\title{
Convection in a rotating laterally-heated annulus
}

\author{
By E. L. KOSCHMIEDER \\ College of Engineering, The University of Texas at Austin, \\ Austin, Texas 78712
}

(Received 28 February 1969 and in revised form 19 March 1971)

The convective motions in a rotating laterally-heated annulus have been studied experimentally. The main feature that differentiates this experiment from others done previously is a rigid lid which is in contact with the fluid. It has been found that the radial temperature differences at which the transition from lower symmetry to the vortex regime occur are different in the cases of positive and negative temperature gradients and that, in particular, the transition is a very strong function of $\Omega$ in the case of a negative temperature gradient. It has been observed further that the persisting vortices which form in the annulus always appear at the inner cylinder, whether the radial temperature difference is increased in a quasi-steady way, the radial temperature difference is applied suddenly while the mean temperature is maintained, the heating or cooling is applied suddenly on either the inner or outer cylinder only, thus changing the mean temperature, or whether the rotation of the annulus is started suddenly with the radial temperature difference applied beforehand. This is true regardless of whether the radial temperature gradient is positive or negative and there has not been a single instance in which a persisting vortex developed at the outer cylinder. Neither this observation nor the dependence of the transition temperature difference on $\Omega$ seems to be in agreement with the current theoretical descriptions of the annulus motions. On the other hand, the appearance of a warm cell, a counter cell and a cold cell in the time-dependent meridional motions observed in the experiments confirms the results of a numerical study of Williams (1967), which studies the symmetric motions in the annulus.

\section{Introduction}

Since the pioneering work of Hide (1958) and the discovery of the lower symmetric regime by Fultz (1959) much work has been done on the convective motions in a rotating laterally-heated annulus. A fairly comprehensive list of these studies appears in McIntyre's (1968) paper. The most interesting feature of the fluid motions in the annulus are the azimuthal 'waves' which appear at certain horizontal temperature differences and rotation rates. The change from axisymmetric flow to waves occurs along a well-defined transition curve and measurements of the transition proved to be reproducible within a small error. However, in the steady wave regime, experiments were not strictly reproducible. To quote Fowlis \& Hide (1965), " $m$ is not uniquely determined by the external parameters", $m$ being the wavenumber. One of the reasons for this could be the 
upper air surface. A rigid lid in contact with the fluid should eliminate the problems which come from the curvature of the upper surface as well as from evaporation, surface tension and wind stress on an air surface. A rigid lid should further make the flow in the annulus nearly symmetric about the plane $z / d=\frac{1}{2}$; under an air surface the flow is not symmetric. There is apparently only one comment in the literature which is relevant to the flow in an annulus under a rigid lid. This is the statement of Bowden \& Eden (1965) that the net relative angular momentum of the fluid "is reduced to approximately zero, when there is a lid in contact with the upper surface". This is an observation made with symmetric flow. The main object of the experiments described below was the investigation of the flow in an annulus with the fluid in contact with a rigid lid. As a by-product of these experiments we have gained some information concerning the development of the waves. Knowledge of the development of the waves should help us to a better understanding of their nature. The time-dependent development of the motion is also of interest when compared with the numerical studies of Williams (1967). Williams' studies are based on very realistic assumptions and provide a fairly extensive insight into the velocity field of axisymmetric motions under an air surface as well as under a rigid lid. As will be seen, some of the features of Williams' computations can be verified experimentally.

\section{Description of the apparatus}

A section through the apparatus used is shown in figure 1. The liquid was contained between two concentric copper cylinders, the outer diameter $2 a$ of the inner cylinder being $48.0 \mathrm{~mm}$, the inner diameter $2 b$ of the outer cylinder being $101.7 \mathrm{~mm}$. The wall thickness was about $4 \mathrm{~mm}$ at the inner cylinder and about $6 \mathrm{~mm}$ at the outer cylinder, the depth $d$ of the liquid was $100 \mathrm{~mm}$ and the lucite top and bottom were of $25 \mathrm{~mm}$ thickness. The dimensions of the annulus were chosen to make this experiment comparable to a previous investigation by Kaiser (1970). The inner cylinder was cooled, in the way shown, by $60 \mathrm{~cm}^{3} \mathrm{~s}^{-1}$ water or more. This arrangement should provide a uniform temperature along the circumference of the cylinder. However, the cooling water warmed up as it moved along the cylinder and exchanged heat with the cylinder. Consequently the temperature of the cylinder wall increased towards the bottom. The variation of the wall temperature was minimal at small radial temperature differences $\Delta T_{r}$ and went up to $1.5 \%$ of $\Delta T_{r}$ at the maximum value of $\Delta T_{r}$. The outer cylinder was heated by about $60 \mathrm{~cm}^{3} \mathrm{~s}^{-1}$ water, running in a $0.8 \mathrm{~mm}$ wide gap along the surface of the cylinder from an upper circular reservoir to a lower circular reservoir, as shown in figure 1. This arrangement should likewise provide a uniform temperature along the circumference, but in this case the wall temperature decreased towards the bottom (up to $1.5 \%$ of $\Delta T_{r}$ ) owing to heat exchange of the water with the cylinder, as before. The arrangement also provided a minimal heat capacity outside the outer cylinder, which is important for experiments with sudden temperature changes. The radial temperature difference $\Delta T_{r}=T_{b}-T_{a}$ applied to the cylinder walls was measured with mercury thermometers placed in the water after it had passed the cylinders. The accuracy of the measurement was 
$\pm 0.1^{\circ} \mathrm{C}$. This measured $\Delta T_{r}$ is identical with the temperature difference applied to the liquid in the annulus gap since the decrease of the temperature in the copper walls is negligible. The whole apparatus was mounted on a turntable with a continuously variable drive. The liquid in the annulus gap was tap water and the motions of the fluid were made visible by aluminium powder suspended in the water with the help of a detergent. Bright areas in the photographs indicate predominantly horizontal motion, while dark areas or lines indicate either vertical or no motion. Pictures were taken with a stationary camera, exposure time was $\frac{1}{250}$ sec.

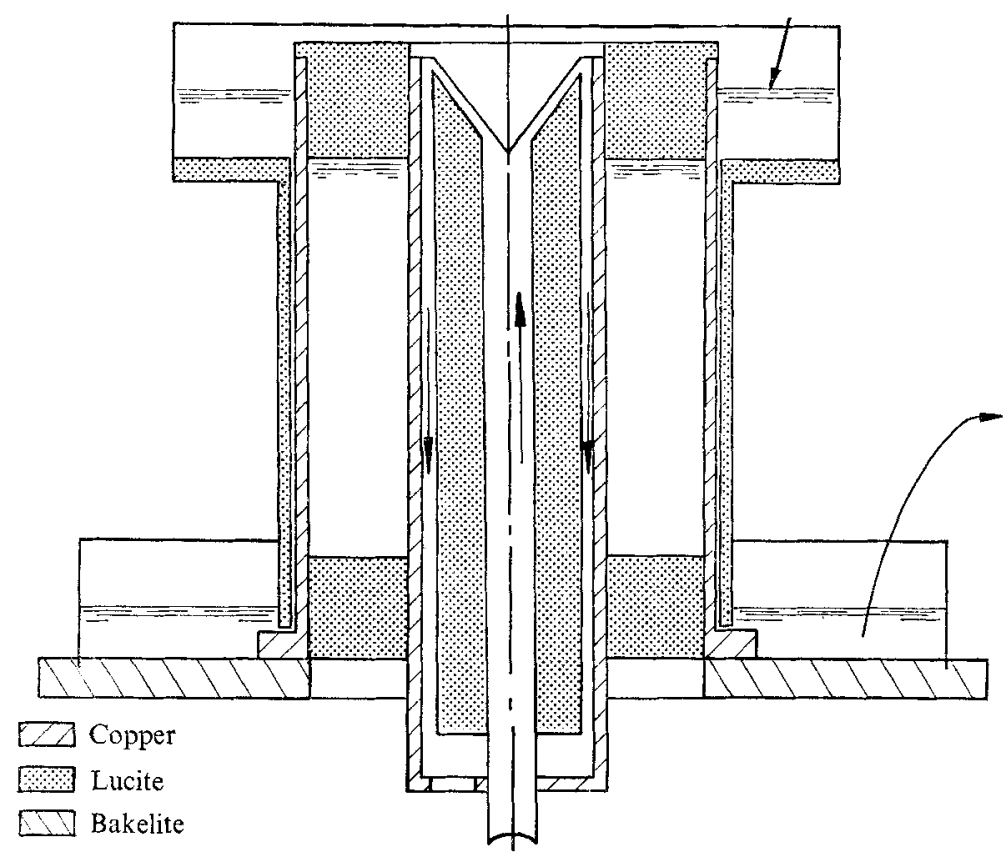

FigURe 1. Section through the apparatus.

\section{Steady experiments}

A number of experiments with steady or quasi-steady wall temperatures were made in order to compare the observations of the fluid motions under a rigid lid with the evidence from the free-surface case. Much of the previous experimental evidence is contained in the transition curves. As an example we reproduce the transition curve measured by Kaiser (1970) (see figure 2). Details of the original have been omitted and new variables introduced. This particular curve has been chosen since the annulus used by Kaiser and the one used here have almost identical dimensions except that the depth of the fluid in his case was $13 \mathrm{~cm}$ as compared with $10 \mathrm{~cm}$ here. Note that at a constant rotation rate and increasing radial temperature difference one passes from the lower symmetric regime through the wave regime to the upper symmetric regime. The wavenumber in the wave regime decreases steadily with increased $\Delta T_{r}$ at constant $\Omega$. A transverse through the wave regime made over $10 \mathrm{~h}$ at $\Omega=2.5 \mathrm{rad} / \mathrm{s}$ with 
the fluid in contact with the lid produced a similar result. First there was lower symmetric flow, then six waves appeared, followed by a one-by-one reduction in the wavenumber with increased temperature difference, till finally at the maximum possible $\Delta T_{r}$ only one wave remained (see figures $3(a)-(d)$, plate 1). On these photographs the direction of rotation is indicated by the arrow on the inner cylinder and the dark line on the lucite lid served as a fiducial mark.

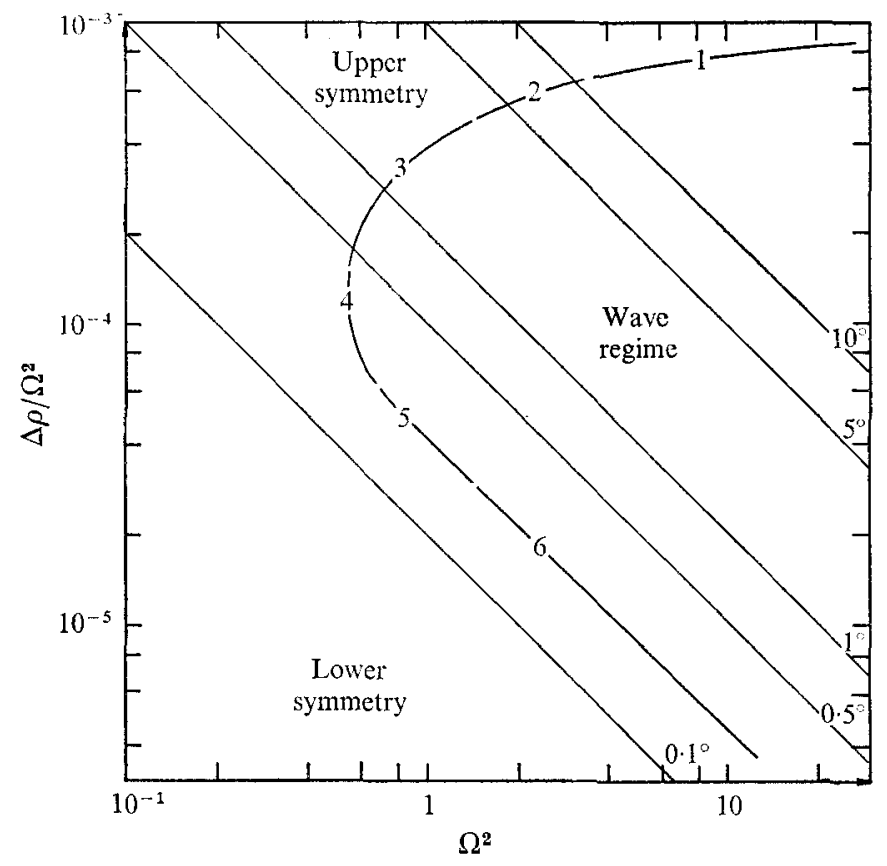

FIGURe 2. Transition curve under an air surface with a positive temperature gradient.

These observations seem to be in qualitative agreement with the air-surface experiments. Also the transition from lower symmetry to the wave regime occurred at a $\Delta T_{r}$ which is compatible with previous measurements. On the other hand, the overall azimuthal motion of the patterns was much slower than under an air surface. With a positive radial temperature gradient this motion is ahead of the rotation (forward). All five-wave patterns were, as far as could be seen, stationary with respect to the rotating cylinders. It took $1030 \pm 20$ s for a four-wave pattern to make $\frac{1}{4}$ of a revolution at a steady $\Delta T_{r}=10 \cdot 4^{\circ} \mathrm{C}$ and under exactly the same conditions, but under an air surface, it took $245 \pm 5$ s for the pattern to make one complete revolution. Increasing the radial temperature difference made the patterns move much faster under the lid; at $\Delta T_{r}=15^{\circ} \mathrm{C}$ three waves revolved once in $690 \mathrm{~s}$ and during that time the amplitude of the pattern oscillated ten times. Such amplitude oscillations have been discussed by Pfeffer \& Fowlis (1968). At $\Delta T_{r}=20^{\circ} \mathrm{C}$ two waves revolved once in $420 \mathrm{~s}$, with twelve amplitude oscillations during that time. Finally, an almost steady onewave pattern needed $159 \pm 3 \mathrm{~s}$ for one complete revolution at $\Delta T_{r}=31^{\circ} \mathrm{C}$, as was determined from 12 consecutive revolutions. With the decrease of the azimuthal 
speed the appearance of the motions under the lid had changed as compared to the air-surface case. An example of an air-surface pattern can be seen in figure $3(e)$. The characteristic wavy jet of fast azimuthal flow is no longer continuous under a lid (figures $3(a)-(d)$ ) and in fact we observe an intensification of the overall azimuthal flow along each of the vortices which extend from the inner cylinder into the fluid. Note that the curved dark lines coming from the inner cylinder in figures $3(a)-(d)$, as well as in figure $3(e)$, indicate vertical (downward) motion. The fluid enters these dark lines almost tangentially and the streamlines are therefore (distorted) spirals. Such a flow has always been called a vortex. So, what we actually see in these photographs as the dominant features are vortices, and we shall henceforth refer to these patterns as vortex patterns. The vortex nature of these patterns will become increasingly clear from the observation of the appearance of the flow with negative temperature gradients, or from the way these patterns develop in time-dependent experiments (see in particular figures $12(f), 13(f)$ and $15(e)$, plates 6,7 and 9$)$ or during intense amplitude oscillations.

A traverse through the wave regime close to the knee of the transition curve at $\Omega=1 \mathrm{rad} / \mathrm{s}$ with the liquid under the lid produced first the lower symmetric flow and then with increased $\Delta T_{r}$ five very weak vortices formed. Further slow heating of the outer wall reduced the number of vortices to four and then three, till finally the flow became symmetric again (upper symmetry). The vortices were weak throughout the entire traverse; they never extended over more than a third of the gap. Close to upper symmetry the amplitudes became so small that the distinction between vortex flow and symmetric flow became rather arbitrary. The evidence at $\Omega=1 \mathrm{rad} / \mathrm{s}$ is again in qualitative agreement with the evidence of the air-surface experiments.

We shall now discuss a traverse through the wave regime when the imposed radial temperature difference is negative, the fluid being in contact with the lid. It is usually assumed that the direction of the radial temperature difference has no significant influence on the transition curve, to quote Fowlis \& Hide (1965), "the most conspicuous effect of reversing the sign of $\Delta T$ is the reversal of the direction of hydrodynamical flow relative to the rotating system ". When at constant $\Omega=2.5 \mathrm{rad} / \mathrm{s}$ the temperature of the inner cylinder was slowly raised, symmetric flow was observed, followed by the transition to vortex flow with eight vortices appearing. Increasing the temperature of the inner wall slowly and steadily resulted in a one-by-one reduction of the vortex number, until at around $\Delta T_{r}=-40^{\circ} \mathrm{C}$ only one vortex remained. Some of the flow patterns are shown in figures $4(a)-(e)$ (plate 2 ) and two corresponding air-surface patterns in figures $5(a)$ and $(b)$ (plate 3 ).

Though these observations seem to be similar to those made with a positive temperature gradient there are several substantial differences. First, the temperature difference at which the transition from lower symmetry occurred was much larger than the transition temperature in the case of a positive $\Delta T_{r}$. This will be discussed in detail later on together with the transition temperature difference for positive $\Delta T_{r}$. We note further that at the transition eight vortices appeared whereas there were six with a positive gradient. Consequently the temperature differences at which the vortex numbers change are different in each 
case. Also, the azimuthal speed of the patterns in the case of negative gradient was different from that for the corresponding positive gradient. This was to be expected from the air-surface experiments. With a negative temperature gradient the vortex pattern should move against the rotation (backwards). Hence it appeared at first to be strange that at $\Delta T_{r}=-4{ }^{\circ} \mathrm{C}$ and $\Omega=2.5 \mathrm{rad} / \mathrm{s}$ the patterns moved very slowly forwards, a four-wave pattern made $\frac{1}{4}$ revolution forward in $3500 \mathrm{~s}$. However, at $\Delta T_{r}=-5^{\circ} \mathrm{C}$ and the same $\Omega$ the motion of the pattern had stopped and at $\Delta T_{r}=-10^{\circ} \mathrm{C}$ it took $1706 \mathrm{~s}$ for a four-wave pattern to make one revolution backwards. At $\Delta T_{r}=-15^{\circ} \mathrm{C}$ it took $1150 \mathrm{~s}$ for one revolution backwards, while the amplitude of the four vortices oscillated eleven times. Under an air surface but with otherwise identical conditions it took $128 \mathrm{~s}$ for one revolution backwards. When the radial temperature difference was increased further, the liquid being in contact with the lid, the azimuthal motion of the patterns slowed down again. At $\Delta T_{r}=-20^{\circ} \mathrm{C}$ it took a three-vortex pattern $969 \mathrm{~s}$ to make $\frac{1}{3}$ revolution backwards and during this time 13 amplitude oscillations occurred. It was then quite startling to note that at $\Delta T_{r}=-25^{\circ} \mathrm{C}(\Omega=2.5$ $\mathrm{rad} / \mathrm{s}$ ) the pattern moved forwards again, making $\frac{1}{3}$ revolution in $8310 \mathrm{~s}$ with 68 amplitude oscillations occurring during this time. At $\Delta T_{r}=-30^{\circ} \mathrm{C}$ two steady vortices made one revolution forwards in $6040 \mathrm{~s}$ and finally a one-vortex pattern needed only $712 \pm 5 \mathrm{~s}$ for one forward revolution at $\Delta T_{r}=-42^{\circ} \mathrm{C}$.

This observation makes it necessary to discuss in more detail the one-vortex pattern as it appears with a negative temperature gradient, an example of which is shown in figure $4(e)$. This pattern has an irregular and unsteady appearance when compared with all the other patterns. It is apparently produced by a large number of small unsteady vortices which pile up at a particular point at the inner cylinder and then form a long train of little vortices moving backwards with the surface flow gradually fading away. This area is followed by a region in which the fluid moves smoothly along the inner eylinder. It is apparently in this section that the little vortices form, also vortices from the end of the train are occasionally carried over the smooth section. The newly formed vortices move backwards, as does the fluid under the lid and pile up before the old vortex bulge. In this way the entire pattern moves forward, against the direction of the flow under the lid. There is nothing in the annulus literature with which to compare this observation. The only experimental evidence concerning a one-vortex pattern is a thermograph record shown in Kaiser's (1970) paper and in this case the temperature gradient was positive. The nature of the one-vortex pattern with a negative gradient seems to be the result of a continuous development. Note that the cores of the vortices in the two-vortex pattern (figure $4(d)$ ) already have a rather irregular structure as compared with the neat vortices in the fourvortex pattern, figure $4(b)$. Actually the development of vortices which pile up on older vortices is quite common in time-dependent experiments and this regeneration of the vortices apparently causes the characteristic broken flanks of the vortices with a positive temperature gradient (see figures $3(a),(b)$ or figure $15(f))$. Also, such pile-ups are frequent with air-surface patterns in which the azimuthal velocities are comparatively large. Note the little vortices on the flanks of the large vortices in figure $3(e)$. 
We shall now discuss the results of a rather extended investigation of the transition from lower symmetry to vortex flow which was prompted by the unusually large transition temperature differences in the experiments described above. It was soon realized that the transition temperature differences for experiments with positive and negative temperature gradients at the same $\Omega$ differ considerably and, furthermore, that with both gradients the transition temperature difference is a function of $\Omega$. The results of these measurements are shown in figure 6 . All transitions have been observed after a very slow increase

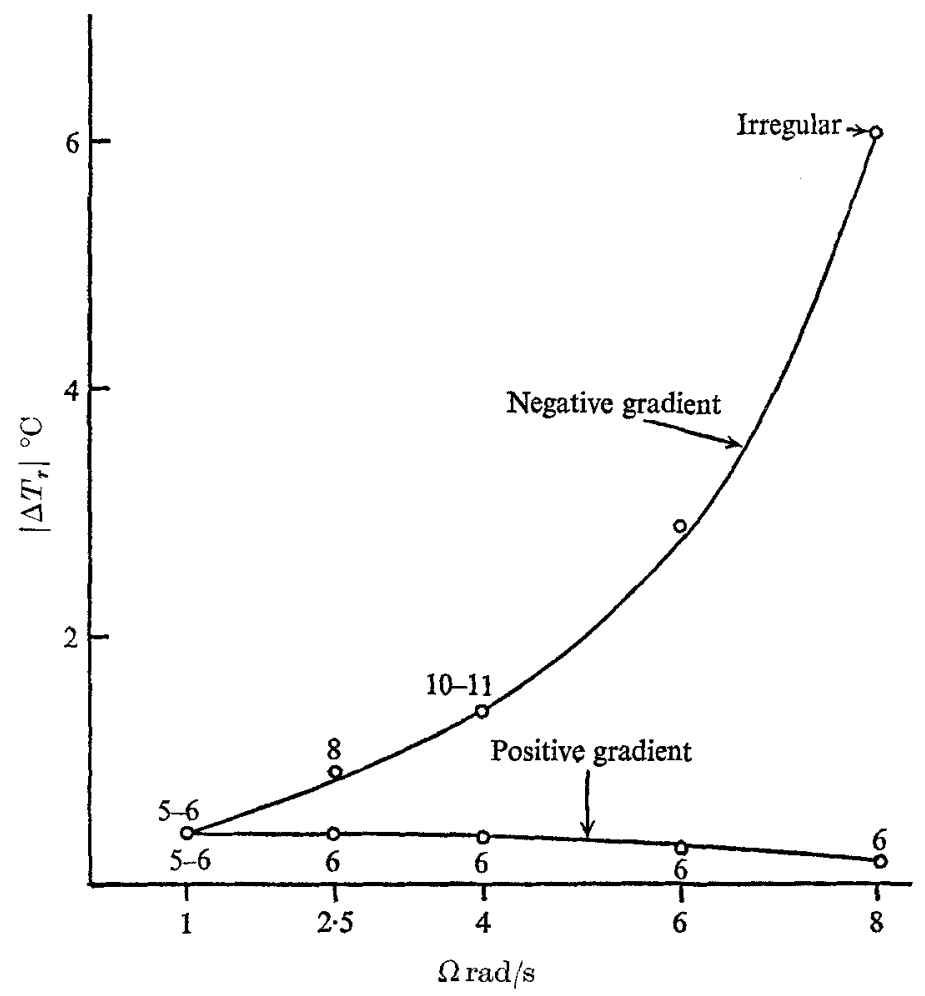

FraUre 6 . Temperature differences for the transition from lower symmetry to vortex flow. The number of vortices formed is indicated at each measured point.

of $\Delta T_{r}$; it took on the average about one hour to come from $\Delta T_{r}=0{ }^{\circ} \mathrm{C}$ to the transition, when the temperature gradient was positive. Also, the temperature was always increased from $\Delta T_{r}=0$ after the fluid had been maintained at $\Delta T_{r}=0$ and constant $\Omega$ for about half an hour. The inaccuracy of the measured $\Delta T_{r}$ is $\pm 0.1{ }^{\circ} \mathrm{C}$ and is due to the variations of the water-bath temperatures. The surprising increase of the transition temperature difference with a negative gradient was first observed by Fultz (1961), who explained the increase in terms of non-linear effects; it has also been observed independently by Kaiser (private communication) in air-surface experiments and by Hide (unpublished). The strong variation of the critical $\Delta T_{r}$ is a good indication that the centrifugal force cannot be neglected in the theoretical studies of annulus motions. In a rotating annulus the centrifugal force tends to set up a centrifugal circulation which 
should move warm light fluid inwards and cool heavy fluid outwards. Note that with a negative temperature gradient the centrifugal circulation is opposite to the thermal circulation, while with a positive temperature gradient the centrifugal circulation is parallel to the thermal circulation. Also, as is shown in figure 6 , with a positive temperature gradient the transition temperature difference decreases with $\Omega$. The decrease is small, yet it amounts ot $50 \%$ of $\Delta T_{r}$ at $\Omega=1 \mathrm{rad} / \mathrm{s}$ and is definitely outside the error of measurements. The $\Delta T_{r}$ plotted in figure 6 are the maximal $\Delta T_{r}$ up to which symmetric flow can be maintained, when the fluid is heated very slowly while rotating at constant $\Omega$. There can be no doubt that vortex patterns can be produced at smaller $\Delta T_{r}$ if either the heating is applied suddenly or the rotation is started suddenly. In such cases the patterns develop in around 15 minutes and persist for about the same time.

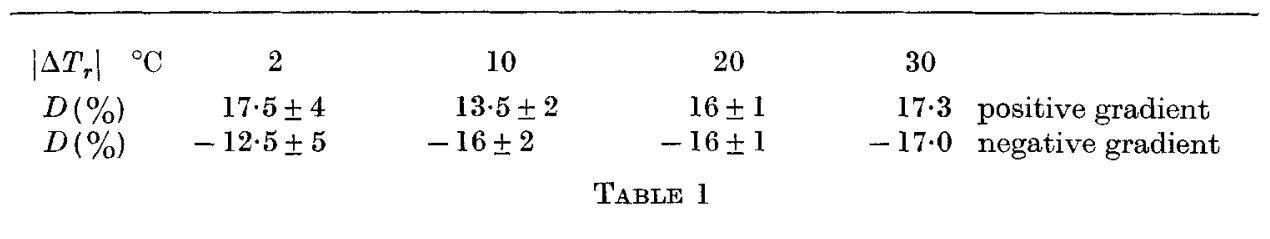

Another set of steady experiments investigated the mean temperature $\bar{T}$ of the fluid in the annulus. The mean temperature is of importance for the timedependent experiments to be described below. Therefore $\bar{T}$ has been measured at several steady $\Delta T_{r}$. If the annulus was rotating, the rotation was then stopped and the lid lifted, while the circulation of the heating and cooling water was continued. Approximately $90 \%$ of the liquid in the gap (about $650 \mathrm{~cm}^{3}$ ) was then immediately siphoned into a beaker which was at the mean wall temperature. It took only a few seconds to perform this operation. The temperature of the liquid in the beaker was then measured. As it turns out, the mean temperature of the liquid differs from the mean wall temperature. The experimental error $\left( \pm 0 \cdot 1^{\circ} \mathrm{C}\right)$ was mainly due to the uncertainty of the mean wall temperature which, in turn, is due to variations of the water temperature in the thermal circulators. Note that $\pm 0 \cdot 1^{\circ} \mathrm{C}$ is at small $\Delta T_{r}$ a substantial fraction of the deviation of the mean fluid temperature $\bar{T}$ from the mean wall temperature $\frac{1}{2}\left(T_{a}+T_{b}\right)=T_{m}$. The deviation $\left(\bar{T}-T_{m}\right) / \Delta T_{r}$ is listed in table 1 for several temperature gradients. Table 1 shows that the deviation is determined by the outer cylinder. A warm outer cylinder produces a positive deviation and with a cold outer cylinder the deviation is negative. The magnitude of the deviation is, within the error of measurement, independent of $\Delta T_{r}$ and independent of the direction of the temperature gradient. The values listed in table 1 are those determined when the rotation rate was $\Omega=2.5 \mathrm{rad} / \mathrm{s}$. When the annulus was at rest the difference $\left|\bar{T}-T_{m}\right|$ was consistently larger than in the rotating case. For example, with $\Delta T_{r}=30^{\circ} \mathrm{C}$ the deviation $D$ was then $18.6 \%$. This is just outside the error of the corresponding value listed in table 1 . The values at smaller $\Delta T_{r}$ fall into the error interval in table 1 and have therefore not been listed. 


\section{Experiments with a suddenly applied positive temperature gradient}

In order to obtain a better insight into the development of the vortices a number of experiments were made in which either the temperature difference across the annulus gap or the rotation rate of the annulus was changed suddenly. There is a variety of ways of changing the temperature difference suddenly. We shall start with a description of the experiments which changed the wall temperatures suddenly while maintaining the mean of the wall temperature. This procedure was suggested by the computations of Williams (1967), who studied the development of symmetric motions in a rotating annulus numerically. He assumed that fluid in the annulus was at a uniform temperature and in rigid rotation at the time $t=0$, at which time, however, the temperature at one wall was $+\Delta T$ higher, and at the other wall $-\Delta T$ lower, than the mean temperature. Williams computes (among others) the streamlines at different times after the sudden heating until the final steady state, see figures 8 and 10. The experiments described below were performed in such a way as to imitate Williams' assumptions experimentally as closely as possible. The experimental procedure was as follows. The annulus was rotated at constant speed while water from one water source was circulated through the inner cylinder as well as along the outer cylinder at a rate of around $60 \mathrm{~cm}^{3} \mathrm{~s}^{-1}$ at each wall. This then defined the mean temperature. When the fluid in the annulus was at the mean temperature and in rigid rotation the wall temperatures were changed by suddenly circulating water from two different water sources through the inner cylinder and along the outer cylinder. The latter two water sources were commercial refrigerated thermal circulators with a control accuracy of $\pm 0 \cdot 1{ }^{\circ} \mathrm{C}$ and supplied water at a rate of around $100 \mathrm{~cm}^{3} \mathrm{~s}^{-1}$. The temperature in these water sources was pre-set in order to raise the temperature of the warm wall by $+\Delta T$ and to lower the temperature of the other wall by $-\Delta T$. Both circulations were switched in a second with the help of eight ball valves, which were connected to the water sources by an intricate plumbing system. This procedure does not, of course, produce an instant change of the wall temperatures by the amount $\Delta T$. The heat capacities of the cylinders, the connecting hoses and the valves produce a temperature variation which approaches its final value exponentially with a very steep initial increase. Note further, that the heat capacities of the inner and outer cylinder are necessarily different. These unavoidable difficulties were noticeable only when the temperature change exceeded $10^{\circ} \mathrm{C}$. In this case the temperature in the water bath serving the outer cylinder was set at $\Delta T+\delta$, the maximal $\delta$ being $0.5^{\circ} \mathrm{C}$. This procedure ensured an initially symmetrical change of the temperature at both walls, while finally changing the mean wall temperature by $\delta$. The final variation of the mean wall temperature appeared to be unimportant.

We start the description of some of the sudden heating experiments with the most simple case, namely, the transition to flow in the upper symmetrical regime. Experiments leading to upper symmetrical flow have been made with temperature changes of $\Delta T= \pm 1{ }^{\circ} \mathrm{C}, \Delta T= \pm 5^{\circ} \mathrm{C}$ and $\Delta T= \pm 10^{\circ} \mathrm{C}$, where $\Delta T= \pm \alpha{ }^{\circ} \mathrm{C}$ stands for a sudden temperature change of $+\alpha^{\circ} \mathrm{C}$ at the outer wall and $-\alpha^{\circ} \mathrm{C}$ at the inner wall while the mean wall temperature remained constant. We can 
describe only one representative example of the transition to upper symmetry here. In this particular case the depth $d$ of the fluid in the annulus was only $50 \mathrm{~mm}$ and the temperature change was $\pm 4.5^{\circ} \mathrm{C}$, while the rotation rate $\Omega$ was $0.7 \mathrm{rad} / \mathrm{s}$. These values were used in order to make this experiment as closely similar to Williams' computations as possible.

The development of the motions after the sudden change of the wall temperatures can be seen on the photographs in figures $7(a)-(d)$ (plate 3). Twelve seconds after the temperature change two symmetric rings can be seen in the annulus (figure $7(a)$ ). The liquid appears fairly dark since it has been in rigid rotation for a while. The aluminium flakes then align vertically and reflect little light.

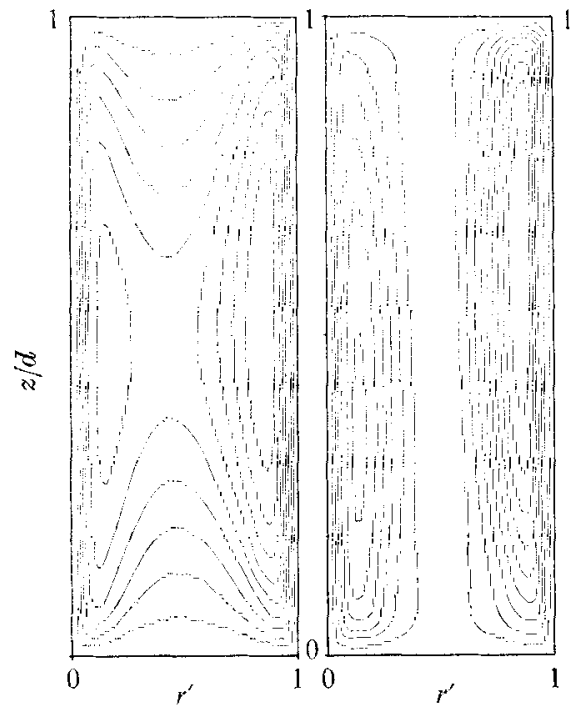

(a)

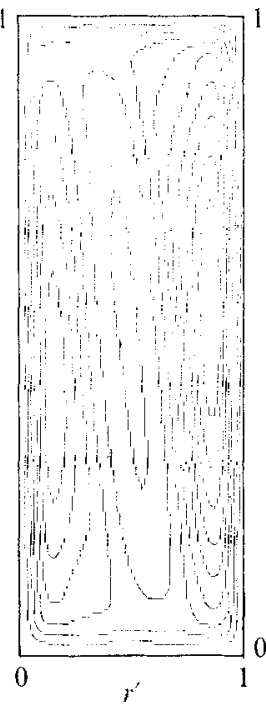

$(c)$

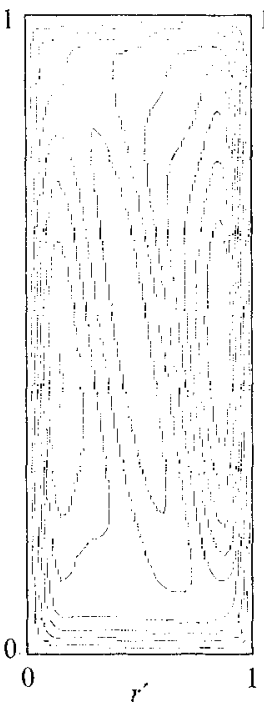

$(d)$

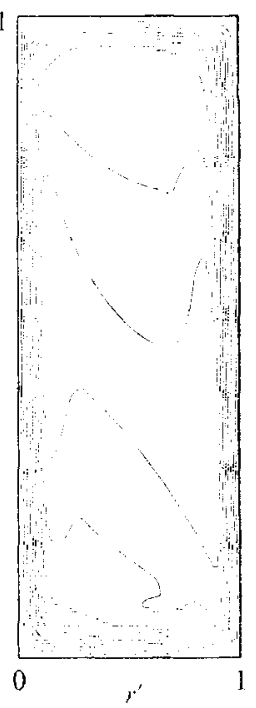

(c)

FIgURe 8. Computed streamlines of non-stationary symmetric flow with a positive temperature gradient. (a) $t=3 \mathrm{~s},(b) t=6,(c) t=24,(d) t=42,(e) t=425$.

The ring about $6 \mathrm{~mm}$ from the outer cylinder marks the interface between the fluid at mean temperature and the 'warm cell' which develops at the outer wall whose temperature has been raised. The faint bright ring at the inner cylinder is the 'cold cell' which develops when the temperature of the inner cylinder is decreased suddenly. At $24 \mathrm{~s}$ (figure $7(b)$ ) the circulations at both walls have intensified markedly and at 31 s (figure $7(c)$ ) the warm cell has expanded along the lid to $6 \mathrm{~mm}$ away from the inner cylinder, the expansion of the warm cell taking place only in the liquid close to the lid. First, an axisymmetric inwardpointing bulge is formed by the warm cell and is rapidly transformed into a wedge with a very sharp tip, the upper side of the wedge being the lid. This can be observed visually and is also predicted by Williams' computations. Thirtyseven seconds after the application of the temperature gradient the warm cell has reached the inner cylinder (figure $7(d)$ ) in order to release its heat there. Symmetric flow follows from then on. 
Let us compare these observations with the results of some computations of Williams, which are presented in figure 8 with the kind permission of Dr Williams. These unpublished computations pertain to an annulus with an inner cylinder of radius $a=34.8 \mathrm{~mm}$, an outer cylinder of radius $b=60.2 \mathrm{~mm}$ and a fluid depth of $50 \mathrm{~mm}$. A rigid lid covers the fluid layer, the rotation rate is specified to be $0.70 \mathrm{rad} / \mathrm{s}$ and the radial temperature difference is $\Delta T_{r}=9^{\circ} \mathrm{C}$. The first graph (figure $8(a)$ ) shows two cells developing from the rotating isothermal liquid. Observed, from above, the corresponding picture would be an annulus of almost uniform brightness if aluminium powder is used for visualization. Figure $8(b)$ shows two separate cells $6 \mathrm{~s}$ after the heating started. Note that the warm cell at the outside turns downward at its inside, while the cold cell turns upwards at its outside. Observed from above, these motions would look quite similar to figure $7(a)$. The next graph (figure $8(c)$ ) would have already appeared as symmetric flow like figure $7(d)$ when observed from above. Note that the shear layer between the warm cell and the cold cell has been replaced by a 'counter cell'. This counter cell is very characteristic and appears on many flow developments to be described later. In figure $8(b)$ the counter cell has moved downward; it dissolves slowly. Finally, a steady flow with minor indications of two cells prevails (figure $8(e))$.

The experiments reported here do not reveal any detail of the flow in the interior of the liquid but a comparison of the results of Williams' computations and these experiments shows both to be in agreement as far as the surface flow is concerned. However Williams' computations indicate that the warm cell arrives earlier at the inner cylinder than the experiments show (less than $24 \mathrm{~s}$ as opposed to $37 \mathrm{~s}$ ). Note that the parameters involved in both cases are the same, except for a difference in the radii of the annulus; Williams used $a=34.8 \mathrm{~mm}$ and $b=60.2 \mathrm{~mm}$ as compared with $a=24.0 \mathrm{~mm}$ and $b=50.8 \mathrm{~mm}$ here. The arrival of the warm cell at the inner cylinder can be observed unambiguously with a very small error $(<1 \mathrm{~s})$. However, different experiments produce slightly different time intervals $\Delta t$ needed for the warm cell to reach the inner cylinder. These differences are probably due to different increases in the wall temperature with time. We assume that the substantial difference between the $\Delta t$ observed and the $\Delta t$ computed is due to the fact that the experiment can only be a poor approximation of the instantaneous wall temperature change on which the computations are based. Some measured $\Delta t$ are listed in table 2 . These time intervals have been observed in the $10 \mathrm{~cm}$ deep annulus. As should be expected, a larger $\Delta T$ shortens $\Delta t$ and the rotation prolongs $\Delta t$. Reducing the depth of the annulus by half while using $\Delta t= \pm 5^{\circ} \mathrm{C}$ increased $\Delta t$ by 5 s. The observations just mentioned are consistent with similar information which can be obtained from experiments with negative temperature gradients (see table 3). They are also consistent with information which follows from sudden-start experiments to be discussed later.

We shall now present an example of the motions after the sudden application of a positive temperature gradient which led to the development of vortices. Such experiments have been made with temperature changes of $\Delta T= \pm 1^{\circ} \mathrm{C}$, $\pm 5^{\circ} \mathrm{C}, \pm 10^{\circ} \mathrm{C}$ and $\pm 15^{\circ} \mathrm{C}$. The rotation rate was usually $2.5 \mathrm{rad} / \mathrm{s}$ though in 
each case control experiments at $\Omega=1.71 \mathrm{rad} / \mathrm{s}$ and $\Omega=3.32 \mathrm{rad} / \mathrm{s}$ were made. It is impossible to discuss each of these cases. We will therefore use as a representative example a case with $\Delta T= \pm 5^{\circ} \mathrm{C}$ and $\Omega=2.5 \mathrm{rad} / \mathrm{s}$ using again the $10 \mathrm{~cm}$ deep annulus. The experimental procedure was as described before. The annulus rotated at constant speed while the outer and inner wall were held at the mean temperature. At the time $t=0$ the temperature of the outer cylinder as raised

$\begin{array}{cccc}\Delta T & \pm 1^{\circ} \mathrm{C} & \pm 5^{\circ} \mathrm{C} & \pm 10^{\circ} \mathrm{C} \\ \Delta t(\Omega=0 \mathrm{rad} / \mathrm{s}) & 32 \mathrm{~s} & 23 \pm 2 \mathrm{~s} & 17 \mathrm{~s} \\ \Delta t(\Omega=0.7 \mathrm{rad} / \mathrm{s}) & 60 \mathrm{~s} & 32 \mathrm{~s} & 25 \mathrm{~s}\end{array}$

TABLE 2

by $5^{\circ} \mathrm{C}$ and the temperature of the inner cylinder lowered by $-5^{\circ} \mathrm{C}$ by circulating water of the respective temperatures along the walls. The temperatures of the heating and cooling water had to be adjusted very carefully in order to obtain a proper counter cell and a symmetric development of the vortices. The development of the motion is shown in figure 9 (plate 4). The first photograph (figure $9(a)$ ) was taken $21 \mathrm{~s}$ after the application of the temperature gradient. The bright ring at the outer cylinder is the warm cell, the narrow ring at the inner cylinder is the cold cell. The counter cell appears between the two rings. Initially the counter cell is wide and the motions there are faint. In particular, the interface between the cold cell and the counter cell is hardly recognizable. The next photograph (figure $9(b)$ ) shows a much more intense counter cell. The width of the counter cell has decreased markedly and the interface to the cold cell has become clearer. (The small non-uniformities of the flow at the outer cylinder in figure $9(b)$ are due to experimental deficiencies and are caused by the liquid which fills the narrow gap between the lucite lid and the outer copper oylinder.) Figure $9(c)$ shows the continuation of this process, the counter cell has been overrun by the warm cell which expands along the lid. Though it is then under the liquid surface, the counter cell could still be observed as a very fine bright band as in figure $9(d)$, where it has already been deformed by the developing vortices. All four vortices are visible at $89 \mathrm{~s}$ in figure $9(e)$ and filled the entire gap at $120 \mathrm{~s}$ (figure $9(f)$ ). This pattern now stabilized quickly. At $t=240 \mathrm{~s}$ a picture of an almost regular steady pattern was taken. The essential reproducible features of this experiment are the warm cell, the counter cell, the cold cell and the appearance of the vortices at the inner cylinder. However, under the same experimental conditions the number of vortices which appeared varied. With $\Omega=2 \cdot 5 \mathrm{rad} / \mathrm{s}$ and $\Delta T= \pm 5{ }^{\circ} \mathrm{C}$ a total of 30 experiments produced four vortices, while 6 experiments produced only three vortices.

The development of the patterns with different positive temperature gradients or different rotation rates was always the same. Initially the three axisymmetric cells appeared, then vortices formed at the inner cylinder. The time needed to establish a steady pattern decreased with increased temperature gradient; at $\Omega=2 \cdot 5 \mathrm{rad} / \mathrm{s}$ it decreased from about $100 \mathrm{~s}$ at $\Delta T= \pm 15^{\circ} \mathrm{C}$ to about $600 \mathrm{~s}$ at $\Delta T= \pm 1^{\circ} \mathrm{C}$. The number of vortices which appeared was sometimes 
reproducible; all nineteen experiments with $\Delta T= \pm 10^{\circ} \mathrm{C}$ and $\Omega=2.5 \mathrm{rad} / \mathrm{s}$ produced three vortices. On the other hand, with $\Delta T= \pm 1^{\circ} \mathrm{C}$ and $\Omega=2.5 \mathrm{rad} / \mathrm{s}$ the number of vortices went up to eight before settling finally at six vortices in the steady state.

\section{Experiments with a suddenly applied negative temperature gradient}

Applying a sufficiently large negative temperature gradient suddenly to the rotating isothermal liquid in the annulus leads to a development of symmetric flow which is analogous to that which has been observed with a positive temperature gradient, as was to be expected from Williams' computations. An example of these computations is shown in figure 10. These unpublished computations

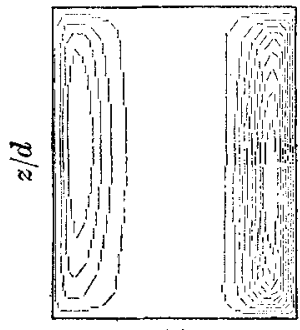

(a)

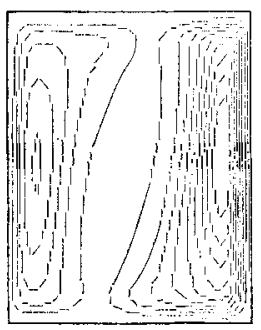

(b)

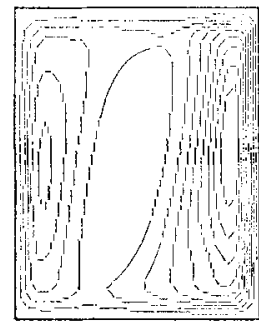

(c)

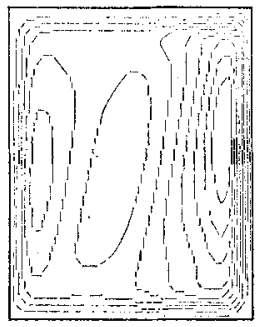

(d)

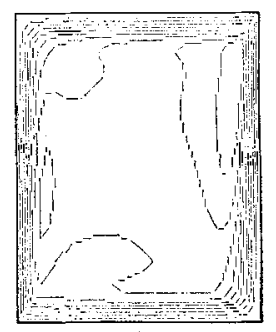

(e)

FIGURE 10. Computed streamlines of non-stationary symmetric flow with a negative temperature gradient. (a) $t=6 \mathrm{~s},(b) t=38,(c) t=48,(d) t=70,(e)$ steady state.

pertain to an annulus with an inner radius $a=20 \mathrm{~mm}$, outer radius $b=50 \mathrm{~mm}$, liquid depth $d=30 \mathrm{~mm}, \Delta T_{r}=-5{ }^{\circ} \mathrm{C}$ and $\Omega=0.8 \mathrm{rad} / \mathrm{s}$. A rigid lid is in contact with the liquid. As before, with a positive gradient, the fluid was supposed to be isothermal at $t=0$, when the wall temperatures were changed instantaneously, in this case by $+2 \cdot 5^{\circ}$ at the inner wall and $-2.5^{\circ} \mathrm{C}$ at the outer wall. Consequently a warm cell now appeared at the inner wall and a cold cell at the outer wall (figure $10(a)$ ). The warm cell expanded along the lid (figure $10(b)$ ) and at $t=40 \mathrm{~s}$ the first streamline extended from the inner cylinder to the outer cylinder along the lid. This would appear as symmetric flow in the experiments. The counter cell can be seen in figure $10(c)$. Though the counter cell is again not visible in experiments which lead to symmetric flow, it is a characteristic feature in the experiments which lead to the development of vortices. There are three cells in figure $10(d)$ and finally weak remnants of two cells in the steady state (figure $10(e)$ ). In order to approximate these computations experimentally the liquid depth in our annulus was reduced to $2.5 \mathrm{~cm}$, so that the aspect ratio in the experiment was almost one, as it is in the computations. When at $\Omega=0.8 \mathrm{rad} / \mathrm{s}$ the wall temperatures were suddenly changed by $\Delta T=\mp 2 \cdot 5^{\circ} \mathrm{C}$, a warm cell and a cold cell appeared; the warm cell expanded perfectly along the lid to reach the outer cylinder after $73 \pm 1 \mathrm{~s}$. Pictures cannot be shown here. Notice that the cell took $73 \mathrm{~s}$ for its expansion, while the computations produced the same result in $40 \mathrm{~s}$. With a positive gradient this was $37 \mathrm{~s}$ as opposed to $24 \mathrm{~s}$, though with a different $\Omega$ and $\Delta T_{r}$. When the same experiment was performed without rotation 
the warm cell arrived at the outer cylinder after only $32 \mathrm{~s}$. Note further that the experiment with $\Omega=0.8 \mathrm{rad} / \mathrm{s}, \Delta T=\mp 2.5^{\circ} \mathrm{C}$ and $d=2.5 \mathrm{~cm}$ led to the formation of five vortices at about $200 \mathrm{~s}$ after the application of the temperature gradient. When the experiments which led to symmetric flow were made with the usual liquid depth in our annulus $(d=10 \mathrm{~cm})$ the warm cell arrived at the outer cylinder after the following time intervals $\Delta t$ (see table 3 ).

$\begin{array}{cccc}\Delta T & \mp 1^{\circ} \mathrm{C} & \mp 5^{\circ} \mathrm{C} & \mp 10^{\circ} \mathrm{C} \\ \Delta t(\Omega=0 \mathrm{rad} / \mathrm{s}) & 42 \pm 2 \mathrm{~s} & 26 \pm 2 \mathrm{~s} & 18 \pm 1 \mathrm{~s} \\ \Delta t(\Omega=0.7 \mathrm{rad} / \mathrm{s}) & 63 \pm 4 \mathrm{~s} & 32 \pm 3 \mathrm{~s} & 25 \mathrm{~s}\end{array}$

TABLE 3

The development of vortices in the case of a negative temperature gradient has been studied with temperature changes of $\Delta T=\mp 1{ }^{\circ} \mathrm{C}, \Delta T=\mp 5{ }^{\circ} \mathrm{C}$ and $\Delta T=\mp 10^{\circ} \mathrm{C}$. The rotation rate was usually $2.5 \mathrm{rad} / \mathrm{s}$ but control experiments with $\Omega=1.71 \mathrm{rad} / \mathrm{s}$ and $\Omega=3.32 \mathrm{rad} / \mathrm{s}$ have also been made. As the characteristic example we have chosen an experiment with $\Delta T=\mp 5{ }^{\circ} \mathrm{C}$ and $\Omega=2.5 \mathrm{rad} / \mathrm{s}$, the depth $d$ being as usual $10 \mathrm{~cm}$ and the liquid being in contact with the lid. The experimental procedure corresponds exactly to the procedure used in the case of positive gradients. Twenty-three seconds after the application of the temperature gradient the first photograph was taken (figure 11 $(a)$, plate 5). There are three axisymmetric cells, at the inside the warm cell which is very bright, then the weak counter cell and at the outside the cold cell. The warm cell and the cold cell intensify at the expense of the counter cell (figure $11(b)$ ) and the counter cell is soon overrun by the warm cell (figure $11(c)$ ). However, the counter cell is still recognizable on figure $11(d)$ as a fine line, deformed to an approximately square configuration. Two vortices have developed on figure $11(e)$ and an almost regular four-vortex pattern could be observed at $t=150 \mathrm{~s}$ (figure $11(f)$ ). We see that the development in this case is very similar to the development of the four-vortex pattern with a positive temperature gradient (figure 9). However, with a negative temperature gradient the vortices develop at the inner cylinder too and turn against the direction of rotation following the azimuthal velocity component of the flow under the lid. Out of 12 experiments with $\Omega=2.5 \mathrm{rad} / \mathrm{s}$ and $\Delta T=\mp 5^{\circ} \mathrm{C}$ only one produced three vortices, the eleven others produced four vortices. With $\Delta T=\mp 1{ }^{\circ} \mathrm{C}$ and $\Delta T=\mp 10^{\circ} \mathrm{C}$ the developments were of the same kind, producing six vortices at $\Delta T=\mp 1^{\circ} \mathrm{C}$ and two or three vortices at $\Delta T=\mp 10^{\circ} \mathrm{C}$. Again, the time interval in which a steady pattern developed decreased markedly with increased $\Delta T$.

\section{Other time-dependent temperature changes}

The set of experiments with suddenly applied temperature gradients which maintain approximately the mean temperature of the fluid described above is only one of several possible methods of making the temperature distribution in the liquid time-dependent. It has, for example, been suggested by Dr M. E. McIntyre 
that some experiments in which the initial temperature of the fluid is nearer to the temperature of the inner wall should be tried. It is comparatively easy to perform an experiment with the entire fluid initially at the temperature of the inner wall and then suddenly increasing the temperature of the outer wall while maintaining the temperature of the inner wall. This procedure does, of course, increase the mean temperature of the fluid. In order to make this kind of investigation complete a number of experiments has been made in which the outer cylinder was either suddenly heated or suddenly cooled, while the temperature of the inner cylinder was maintained and vice versa, the inner cylinder being: either suddenly heated or suddenly cooled while the temperature of the outer cylinder was maintained.

We shall describe two representative examples of these experiments. First, for sudden heating of the outer wall, $\Delta T_{0}=+10^{\circ} \mathrm{C}, \Omega=2.5 \mathrm{rad} / \mathrm{s}$, the procedure was as follows. Water from one water source was circulated along the inner and the outer cylinder while the annulus was at a constant rotation rate. Thus the liquid in the annulus gap was isothermal and in rigid rotation when the temperature of the outer cylinder was changed suddenly by circulating water with a different temperature along the outer wall. The temperature of the inner cylinder was maintained, however, by continuing the circulation through it at the original temperature. The development of the motion is shown on the photographs in figure 12 (plate 6). Thirty-two seconds after the increase of the temperature of the outer wall a strong warm cell has developed (figure $12(a)$ ) with a counter cell inside it. The warm cell soon overruns the counter cell (figure 12(b)). The fluid inside the warm cell is comparatively dark on the photograph since there is little or no motion there and the aluminium flakes are therefore aligned vertical, producing little reflexion. On the next photograph (figure $12(c)$ ) the warm cell has extended up to the inner cylinder, except for a very narrow almost square area out of the corners of which the vortices developed (figures $12(d)$ and $(e)$ ). Figure $12(f)$ shows four vortices extending over the entire gap $180 \mathrm{~s}$ after the experiment was started. Finally, four fairly steady vortices were observed at $t=300 \mathrm{~s}$.

For a comparison figure 13 (plate 7) shows the development of the motion when the inner cylinder is suddenly cooled with $\Delta T_{i}=-10^{\circ} \mathrm{C}, \Omega=2.5 \mathrm{rad} / \mathrm{s}$. The experimental procedure corresponds exactly to the procedure in the suddenheating experiment just described. As can be seen on figure $13(\alpha)$, an intense cold cell formed $51 \mathrm{~s}$ after the temperature of the inner cylinder was lowered by $10^{\circ} \mathrm{C}$. The interface between the cold cell and the rest of the liquid is less distinct on the next photograph, figure $13(b)$. The cold cell then deforms to a square configuration out of the corners of which the vortices developed (figures 13(c)- $(e)$ ). At $t=117 \mathrm{~s}$ the vortices extended over the entire gap (figure $13(f)$ ) and $6 \mathrm{~s}$ later they were of the typical hook form, one side extending from the inner cylinder to the outer cylinder. Perpendicular to this flank was a section of approximately the same length parallel to the outer cylinder, the top of this section still showing the characteristic curl. Finally, at $t=600 \mathrm{~s}$ a regular steady four-vortex pattern was observed. The number of vortices produced through such sudden heating or sudden cooling was again not strictly reproducible, twenty-three experiments with $\Omega=2 \cdot 5 \mathrm{rad} / \mathrm{s}$ and $\Delta T_{0}=10^{\circ} \mathrm{C}$ or $\Delta T_{i}=-10^{\circ} \mathrm{C}$ produced four vortices while 
seven experiments produced only three vortices. In general, the development of the patterns was more symmetric than in the case of suddenly applied temperature gradients which maintained the mean wall temperature. Sudden-heating and sudden-cooling experiments have also been made with temperature changes of $1{ }^{\circ} \mathrm{C}$ and $5^{\circ} \mathrm{C}$ on either side, all at $\Omega=2 \cdot 5 \mathrm{rad} / \mathrm{s}$. These experiments produced basically the same result; either a warm cell or a cold cell formed, followed by the development of vortices at the inner cylinder. With a free upper surface this was also true in the case of a suddenly heated outer wall. On the other hand, under a free surface no cold cell could be observed when the inner cylinder was cooled suddenly. However in this case the vortices definitely formed at the inner cylinder too. Analogous experiments with sudden heating of the inner cylinder or sudden cooling of the outer cylinder with the liquid in contact with the lid produced again either a warm cell at the inner cylinder or a cold cell at the outer cylinder which expanded with time over the gap. In either case the vortices formed at the inner cylinder once more. Pictures cannot be shown here.

\section{Sudden-start experiments}

The rotation rate is the second fundamental parameter which is involved in the annulus experiments and which can be made time-dependent. No modification of the experimental set-up is needed to perform a sudden-start experiment. First, a proper steady radial temperature difference $\Delta T_{r}$ is established across the gap in the stationary apparatus. Then at the time $t=0$ the motor is switched on to turn with a pre-selected rotation rate. Steady rotation is obtained to within a second. The fluid in the annulus, however, adjusts only slowly to the rotation and a characteristic and reproducible flow development can be observed. It is quite surprising that the developments after a sudden start correspond to a large measure to the flow developments in the experiments with a suddenly applied temperature gradient. The description of some of the sudden-start experiments begins with the most spectacular case, namely the development of symmetric flow as shown in figure 14 (plate 8). A different perspective is advantageous for these photographs. In this experient $\Omega \mathrm{was} 1 \cdot 13 \mathrm{rad} / \mathrm{s}$ and $\Delta T_{r}=9^{\circ} \mathrm{C}$, these parameters correspond to upper symmetry under steady conditions (figure 2). The fluid is in contact with the lucite lid. Immediately after the start of the rotation an intense mixing of the fluid took place but no characteristic feature developed during this time. The mixing gave way to a more regular flow which gradually developed into apparently rigid rotation. When the flow appeared to be close to rigid rotation at about $30 \mathrm{~s}$ after the start of the rotation a weak dark ring appeared adjacent to the outer cylinder. The dark ring moved inwards and became very distinct. Thirteen seconds after its appearance it looked like figure $14(a)$. The dark ring is the interface between an axisymmetric cell, which forms at the outer cylinder (the 'warm cell'), and the rest of the water around the inner cylinder. We observe vertical motion at the interface, hence there is little reflexion of light and consequently the interface appears dark on the photograph. Actually fluid which moves upwards along the outer cylinder reverses its direction in the warm cell and moves downwards at the interface. Closer inspection of 
the photograph reveals that under the lid there is a small triangular gap filled with stagnant liquid, between the warm cell and the rest of the fluid. The next photograph (figure 14(b)) shows that the interface has moved a little inwards and that there is an inward slope of the upper end of the warm cell. The warm cell is now set to expand along the lid towards the inner cylinder. Figure 14(c) shows this expansion happening. There is no longer the triangular gap at the interface and visual observation of the flow shows that the inward tip of the warm cell is only a very few $\mathrm{mm}$ deep. Finally, figure $14(d)$ shows the flow after the warm cell has arrived at the inner cylinder, however, under the surface of the liquid a remnant of the warm cell can still be seen.

There is a very close resemblance between this experiment and experiments with suddenly applied positive temperature gradients. This resemblance is obvious from an comparison of figure 7 and figure 14 . The experiments show that the time interval between the appearance of the warm cell and the arrival of the warm cell at the inner cylinder is the same in the case of sudden temperature gradients and sudden starts, if the parameters in both cases are the same. We therefore explain the development of the flow in the sudden-start experiments in the following way. After the start of the rotation the fluid in the annulus is soon mixed more or less completely which means it will be at approximately the mean wall temperature at the end of the mixing period. The fluid then approaches rigid rotation and finds itself in a situation which corresponds to the assumptions of the computations of Williams', namely it will be an isothermal rotating liquid bounded by a warmer $\left(+\frac{1}{2} \Delta T_{r}\right)$ outer cylinder and a colder $\left(-\frac{1}{2} \Delta T_{r}\right)$ inner cylinder. Consequently a warm cell should form, likewise a cold cell, which is indicated in figures $14(a)$ and $(b)$ by the dark circular area around the inner cylinder. The warm cell should expand as the fluid at the outer cylinder adjusts to the presence of the warm outer wall and finally the warm cell should turn over to the cold inner cylinder just as observed. The same experiment made with an air surface leads to a rather different result. Just after the start a very intense cell forms at the inner cylinder. The motion in this cell is upwards at the inner cylinder and it extends to about $\frac{1}{3}$ gap width, then shrinks slowly and gives way to symmetric flow. No warm cell ever appears at the surface. Finally, vortices form out of apparently symmetric flow at the inner cylinder.

As the development of the symmetric flow is strikingly similar to the corresponding sudden-heating experiment, so is the development of vortex flow after a sudden start similar to the development of vortices after sudden heatings. Sudden-start experiments which lead to vortex flow have been made with radial temperature differences of $2,10,20$ and $30^{\circ} \mathrm{C}$ at $\Omega=2 \cdot 5 \mathrm{rad} / \mathrm{s}$ as well as at $\Omega=1.71 \mathrm{rad} / \mathrm{s}$ and $\Omega=3.32 \mathrm{rad} / \mathrm{s}$. As a representative example we shall discuss the formation of four vortices when $\Delta T_{r}=10^{\circ} \mathrm{C}$ and $\Omega=2 \cdot 5 \mathrm{rad} / \mathrm{s}$. About $35 \mathrm{~s}$ after the start the warm cell appeared at the outer wall, while the rest of the fluid seemed to be in uniform rotation. The warm cell moved slowly inwards (figure 15 $(a)$, plate 9) while the fluid inwards from the warm cell began to subdivide into a cold cell and a counter cell. While, for example, at $\Delta T_{r}=2{ }^{\circ} \mathrm{C}$ a perfect subdivision into cold cell and counter cell occurred, at $\Delta T_{r}=10^{\circ} \mathrm{C}$ the axial symmetry of the motion broke up in order to form the outline of a 
square (figure $15(b)$ ), out of the corners of which the vortices emerged (figure $\mathbf{1 5}(c)$ ). The amplitudes of the vortices increased rapidly (figure $\mathbf{1 5}(d)$ ) and soon extended over the entire gap (figure $15(e)$ ). At $t=158 \mathrm{~s}$ a picture of an almost regular four-vortex pattern was taken (figure $15(f)$ ). An amplitude oscillation of the pattern begin immediately after the picture was taken. As we can see, the development after this sudden start is basically of the same kind as the development discussed in context with figure 9 . Also, the number of vortices formed was not reproducible. Out of twenty-five sudden-start experiments with $\Omega=2.5 \mathrm{rad} / \mathrm{s}$ and $\Delta T_{r}=10^{\circ} \mathrm{C}$ fifteen experiments produced three vortices and ten experiments produced four vortices. That means that three vortices were preferred in the sudden-start experiments, while the formation of three vortices was an exception in the corresponding sudden temperature experiments (six out of thirty-six experiments).

We shall now discuss a new feature which appeared gradually in the suddenstart experiments when the radial temperature difference was increased above $10^{\circ} \mathrm{C}$. With $\Omega=2.5 \mathrm{rad} / \mathrm{s}$ and $\Delta T_{r}=30^{\circ} \mathrm{C}$ a perfect warm cell appeared at first as is usual after a sudden start, see figure $16(a)$ (plate 10) taken $21 \mathrm{~s}$ after the start. However, within a few seconds the interface between the warm cell and the rest of the body of water took on a wavy configuration, see figure $16(b)$ taken at $t=29 \mathrm{~s}$. The amplitude of these waves, which will tentatively be called 'short waves', increased a little until $t=31 \mathrm{~s}$. They then faded away rapidly (figure $16(c)$ ). The next feature to appear was a transient five-wave pattern (figure $16(d)$ ). This pattern persisted until about $60 \mathrm{~s}$ and then gave way to a two vortex pattern at $t=68 \mathrm{~s}$ (figure $16(e)$ ). The two vortices solidified quickly and looked like figure $16(f)$, taken $86 \mathrm{~s}$ after the sudden start. With $\Delta T_{r}=30^{\circ} \mathrm{C}$ and this $\Omega$ two vortices had to be expected. The two-vortex pattern shown in figure $16(f)$ began immediately with an amplitude oscillation, as is normal for the twovortex pattern with steady $\Delta T_{r}$ and $\Omega$. The appearance of the short waves as well as the transient five waves are strictly reproducible. Likewise the number of the short waves (eleven in figure $16(b)$ ) is reproducible within \pm one wave. Though the short waves will most likely be of little importance to the theoretical investigation of the annulus motion, they are interesting as such and we shall try to give a qualitative explanation. If at the same $\Delta T_{r}$, say $\Delta T_{r}=30^{\circ} \mathrm{C}$, sudden starts to increasingly fast rotation rates are made, then one observes that the short waves appear sooner after the sudden start and that the amplitudes of the waves increase. At the inside of the warm cell there is a strong shear of the vertical (downward) velocity component which normally produces the counter cell. At large $\Omega$ we have apparently, at the same place, a strong azimuthal shear, the fluid in the warm cell at the outer cylinder moving approximately with the azimuthal velocity of the cylinder, the fluid inwards from the cell lagging behind. The combination of vertical and azimuthal shear apparently produces the short waves which are vortices rather than waves. In this context it is perhaps pertinent to note that even during the final formation of the vortices in figures $16(e)$ and $(f)$ the fluid at the inner cylinder lags behind the motion of the inner cylinder. This is evident from the backward motion of the origin of the vortices at the inner cylinder (figures 16(e), $(f)$ ). (This backward motion continued beyond the 
fiducial mark till about $t=100 \mathrm{~s}$.) This backward motion of the origin of the vortices is a common feature of all sudden-start experiments, see also figure 15 . It does not, however, occur with the sudden-heating experiments. Another interesting phenomenon is the transient five waves shown in figure $16(d)$. Note that in an annulus of that size and with that $\Delta T_{r}$ and $\Omega$ five waves do not normally appear. However, five waves would be possible with these parameters if the outer diameter of the annulus were substantially reduced. An effective reduction of the outer diameter of the body of fluid is apparently brought about by the warm cell. The motion is apparently at first restricted to the fluid inwards from the warm cell, consequently more waves can form. This is also a common feature of sudden starts with small $\Delta T_{r}$, when usually more vortices form than stay.

We shall finally discuss sudden-start experiments with a negative temperature gradient. Such experiments have been made with radial temperature differences of $-2,-10,-20,-30$, and $-40^{\circ} \mathrm{C}$, mainly at $\Omega=2 \cdot 5 \mathrm{rad} / \mathrm{s}$. It was expected that in these cases a warm cell would form and expand from the inner cylinder. Surprisingly no warm cell ever appeared in these experiments. There was again a short mixing period just after the start. The flow under the lid then remained uniform except right at the inner cylinder where weak small irregular motions developed out of which the vortices formed; at large $\Delta T_{r}$ apparently through accumulation of smaller vortices. Pictures cannot be shown here. Also, no short waves ever appeared in these experiments. The number of vortices was again not reproducible; out of sixteen sudden starts with $\Delta T_{r}=-10^{\circ} \mathrm{C}$ and $\Omega=2.5 \mathrm{rad} / \mathrm{s}$ eight ended with four vortices, the other eight with three vortices. Though no warm cell appeared after such sudden starts, an intense warm cell appeared at the inner cylinder after each sudden stop of the rotation, provided the radial temperature difference was negative. This warm cell expanded over the gap in a perfect symmetrical form if the flow in the rotating annulus had previously been axisymmetric. The very striking failure of the warm cell to appear after sudden starts with negative $\Delta T_{r}$ is probably due to insufficient mixing after the start. A uniform temperature distribution in the fluid is never even approached, as it apparently is after sudden starts with positive $\Delta T_{r}$. With a negative gradient the initial state before the start has stratified warm light fluid at the inner cylinder and stratified cold heavy fluid at the outer cylinder. This is a stable density distribution as far as the centrifugal force is concerned. Visual observation indicates that the mixing in this case is indeed less thorough than in the case of a positive temperature gradient.

\section{Conclusions}

The experiments described above seem to have brought forward four essential results. The first is the good qualitative agreement in the meridional velocity field between the computations of Williams (1967) and the experiments with suddenly applied temperature gradients which maintain the mean wall temperature.

On the other hand, there are three facts which the theories describing the 'waves' in the annulus apparently do not predict, namely (i) the strong depen- 
dence of the transition temperature difference on $\Omega$ in the case of a negative temperature gradient; (ii) the reversal of the motion of the patterns from backward to forward with respect to the cylinder if the negative temperature is sufficiently large and finally (iii) the fact that in the quasi-steady as well as in the time-dependent experiments the vortices in the annulus always develop at the inner cylinder. This occurs regardless of whether the temperature gradient is positive or negative and regardless of whether the initial fluid temperature equals that of the outer wall or that of the inner wall, or is somewhere in between. By vortices we mean large amplitude non-axisymmetric disturbances that persist indefinitely. Visually different non-axisymmetric disturbances which we have called 'short waves' develop under particular conditions inwards from the warm cell. They do not persist in time.

In particular the observation that the persisting vortices always form at the inner cylinder seems to be crucial. There has not been a single exception from this elementary rule in 437 experiments. This observation indicates that the temperature distribution in the liquid is not the primary cause of the formation of the vortices. It clearly shows that the inner cylinder is of particular significance, and it brings the very large lateral walls to our attention; in these deep annulus experiments these may well be a dominant feature. It might be that the vortices are caused by an instability of the boundary layer at the inner cylinder. A theoretical study of the stability of the lateral boundary layers has yet to be made.

This work was begun during 1968 at the University of Oklahoma where the friendly interest and support of Professor Y. Sasaki and Professor E. M. Wilkins were greatly appreciated. Thanks are due also to Dr E.W. Friday, Dr J.A.C. Kaiser and Dr G.P. Williams for very important help and information and for permission to use figures 2,8 , and 10 . The work was supported by NSF Grant GA-3128 and ESSA Grant E-22-180-68G, during 1968, and by NSF Grant GA-11484 during 1970.

\section{REFERENCES}

Bowden, M. \& Edev, F. H. 1965 J. Atmos. Sci. 22, 185.

Fowlis, W. W. \& Hide, R. 1965 J. Atmos. Sci. 22, 541.

Futtz, D. 1959 Met. Mono. 4, no. 21.

Fultz, D. $196 \mathrm{I}$ Advances in Geophys. 7, 39.

Hide, R. 1958 Phil. Trans. Roy. Soc. A 250, 441.

Karser, J. A. C. 1970 Tellus, 22, 275.

McINTyRe, M. E. 1968 J. Fluid Mech. 32, 625.

Pfeffer, R. L. \& Fowlis, W. W. 1968 J. Atmos, Sci. $25,361$.

Willtams, G. P. 1967 J. Atmos. Sci. 24, 144, 162. 